\title{
Electronic properties of Manganese impurities in Germanium
}

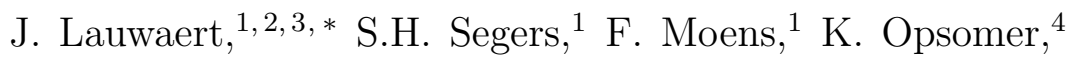 \\ P. Clauws,${ }^{1}$ F. Callens, ${ }^{1}$ E. Simoen,${ }^{1,4}$ and H. Vrielinck ${ }^{1}$ \\ ${ }^{1}$ Department of Solid State Sciences, Ghent University, \\ Krijgslaan 281-S1, 9000 Gent, Belgium \\ ${ }^{2}$ Department of Industrial Technology and Construction, \\ Valentin Vaerwyckweg 1, 9000 Gent, Belgium \\ ${ }^{3}$ Liquid Crystals and Photonics Group, Ghent University, \\ Sint-Pietersnieuwstraat 41, 9000 Ghent, Belgium \\ ${ }^{4}$ IMEC, Kapeldreef 75, 3001 Leuven, Belgium
}

(Dated: February 14, 2015)

\begin{abstract}
The electronic properties of manganese in crystalline germanium have been investigated by means of deep level transient spectroscopy (DLTS). Mn was diffused in the material by a thermal treatment at $700^{\circ} \mathrm{C}$. Next to the deep levels of nickel and copper, which are known contaminants in Ge treated at high temperature, three not previously reported levels were observed. These two hole and one electron traps, with apparent energy level positions at $\mathrm{E}_{\mathrm{V}}+0.136 \mathrm{eV}, \mathrm{E}_{\mathrm{V}}+0.342 \mathrm{eV}$ and $\mathrm{E}_{\mathrm{C}}-0.363 \mathrm{eV}$, were assigned to substitutional Mn. The analysis of the carrier capture cross-sections, the absence of field-assisted emission and the observation of the $\mathrm{Mn}^{2-}$ electron paramagnetic resonance spectrum in n-type Ge:Mn at low temperature are all compatible with Mn introducing two acceptor and one donor levels in the band gap of Ge.
\end{abstract}

PACS numbers: $71.55 . \mathrm{Cn}$

*Electronic address: Johan. Lauwaert@UGent. be 


\section{INTRODUCTION}

The significantly higher carrier mobility of germanium relatively to silicon has introduced a renewed interest to apply germanium in advanced electronic devices. This revival has lead to a strong resurgence of defect studies in germanium in the recent years.[1-3] Since the discovery by Park et al.[4] that $\mathrm{Ge}_{1-\mathrm{x}} \mathrm{Mn}_{\mathrm{x}}$ alloys exhibit ferromagnetic behavior, germanium doped with manganese has been investigated for its application in spintronic devices. Tian et al.[5] produced $\mathrm{p}-\mathrm{Ge}_{1-\mathrm{x}} \mathrm{Mn}_{\mathrm{x}} / \mathrm{i}-\mathrm{Ge}$ epitaxial heterojunctions that possess magnetic field tunable rectifying properties with molecular beam epitaxy. Recently the attractiveness of this technology was further increased as it was shown that these ferromagnetic contacts can be fabricated on the active regions of Ge-based devices by means of a solid state reaction that is compatible with CMOS technology.[6] The origin of the ferromagnetism has been assigned to the formation of $\mathrm{Mn}_{5} \mathrm{Ge}_{3}$ crystallites and $\mathrm{Mn}$ precipitates in the structure, which were not created in boron doped reference specimens.[7] Although the phase of interest, $\mathrm{Mn}_{5} \mathrm{Ge}_{3}$, is easily produced, it is difficult to keep it stable during subsequent device fabrication process steps. It is therefore not easy to avoid Mn trace contamination in the i-Ge layer. Like other transition metal impurities, Mn may be expected to introduce several deep levels in the band gap of Ge, which even at very low concentrations have a severe impact on the electronic properties of the host.

In the four decades after its introduction, deep-level transient spectroscopy (DLTS) has developed into the most powerful and reliable technique for characterizing electrically active defects in semiconductors.[8-13] In view of the above-mentioned applications, it is rather surprising that Mn-related defects in Ge have so-far not been studied with DLTS. The present knowledge of Mn-defect levels in Ge relies on temperature-dependent resistivity measurements by Woodbury and Tyler,[14] performed on Ge crystals doped with Mn in the melt. These experiments revealed two defect levels at $\mathrm{E}_{\mathrm{V}}+0.16 \mathrm{meV}$ (in p-Ge:Mn) and $\mathrm{E}_{\mathrm{C}}-0.37 \mathrm{meV}$ (in n-Ge:Mn). In analogy with results for $\mathrm{Fe}$, Co and $\mathrm{Ni}$, these observations were attributed to a double acceptor character of the Mn dopant. This was confirmed by Electron Paramagnetic Resonance (EPR) measurements, wherein the spectrum of the $\mathrm{Mn}^{2-}$ double acceptor state $\left(3 \mathrm{~d}^{5}\right.$ electron configuration, electron spin $\mathrm{S}=5 / 2$, nuclear spin $\mathrm{I}=5 / 2)$ was identified.[15]

More recently, spectroscopic studies of Ge:Mn have been performed aiming at elucidating 
the geometry of Mn-related defects in Ge. As suggested by Decoster et al.[16] the magnetic behavior of a transition metal impurity strongly depends on its location in the lattice. They concluded from electron emission channeling experiments that a substantial fraction of the Mn occupies the bond-centered site. On the other hand, extended x-ray absorption fine-structure experiments performed after ion implantation at different substrate temperature from room temperature to $300^{\circ} \mathrm{C}$ as well as after magnetron cosputtering, showed evidence for the presence of Mn atoms on the substitutional lattice site, $[17,18]$ consistent with the substitutional $\mathrm{Mn}^{2-}$ impurity observed with EPR by Watkins[15] and the acceptor character of this dopant observed in resistivity studies. In this work the results of DLTS measurements on n- and p-type Ge doped with Mn by metal evaporation and indiffusion are presented. These experiments revealed one donor and two acceptor levels: $\mathrm{Mn}^{+/ 0}$ at $\mathrm{E}_{\mathrm{V}}+136 \mathrm{meV}, \mathrm{Mn}^{0 /-}$ at $\mathrm{E}_{\mathrm{V}}+342 \mathrm{meV}$, and $\mathrm{Mn}^{-/ 2-}$ at $\mathrm{E}_{\mathrm{C}}-295 \mathrm{meV}$.

\section{EXPERIMENTAL}

The starting materials consisted of p-type Ge wafers with a Ga shallow acceptor concentration of $6 \times 10^{14} \mathrm{~cm}^{-3}$ and n-type Ge wafers with an Sb shallow donor concentration of $2 \times 10^{14} \mathrm{~cm}^{-3}$, both supplied by Umicore Electro-Optic Materials. After an etch in $\mathrm{HNO}_{3}$ : $\mathrm{HF}(3: 1)$, Mn dots were deposited on the samples by evaporation of $99.995 \%$ pure Mn, after which a thermal treatment was given. This consisted of heating from room temperature to $700^{\circ} \mathrm{C}$, at which temperature the samples were kept during 10 minutes, before turning off the furnace. The duration of the total process was about 30 minutes. After this thermal treatment, the residual metallic Mn was etched away using a similar etch as before the deposition. On one n-type sample, this additional etch was skipped and replaced by an additional thermal treatment $\left(400^{\circ} \mathrm{C}\right.$ during 20 minutes). This resulted in a metallic manganese-germanide layer, which served as Schottky contact for DLTS. The other samples were prepared for DLTS measurements by evaporating In (on p-type Ge) or Au (on n-type Ge) to form a Schottky junction. The In ( $\mathrm{Au})$ dots were deposited both on and in between places where originally Mn had been deposited. In this way, the effect of the thermal treatment (not related with Mn-doping) could be evaluated on reference diodes. Ohmic contacts were prepared using In-Ga eutectic and In foil. Capacitance DLTS measurements 
were performed with a Fourier transform instrument (Phystech FT1030) equipped with a Boonton 72B capacitance meter with an AC test signal of $1 \mathrm{MHz}$. The sample was placed in a Heraeus contact gas liquid He cryostat, shielded from the environmental black-body radiation by four heat shields. In the DLTS measurements, capacitance transients were recorded during a window-time $T_{w}$ at reverse voltage $\mathrm{V}_{R}$ after a filling pulse (100 ms) to $\mathrm{V}_{P}\left(\mathrm{~V}_{R}<\mathrm{V}_{P}\right)$ and a waiting time $\mathrm{t}_{0}$. EPR measurements were performed at $34 \mathrm{GHz}$ on a Bruker ElexSys E500 EPR-ENDOR spectrometer equipped with an Oxford CF935 liquid helium flow cryostat.

\section{RESULTS}

Figure 1 (a) and (b) show the DLTS spectrum of Mn diffused n- and p-type Ge respectively. In n-type a single band Mn-E1 is observed and in p-type two so far not reported peaks Mn-H1 and Mn-H2 were observed. For these three peaks, a similar diffusion profile was measured. The presence of these three levels was confirmed in a measurement on the n-germanide sample, pulsed from $\mathrm{V}_{R}=-1 \mathrm{~V}$ towards $\mathrm{V}_{P}=1 \mathrm{~V}$, where the Mn-H1 and Mn-H2 peaks appear as minority carrier peaks and the Mn-E1 peak as positive peak. The positions of the three peaks are nearly independent of the electric field. Since in the p-type specimen contamination of $\mathrm{Cu}$ and $\mathrm{Ni}[19,20]$ was present, for comparison a second DLTS measurement was performed on the same sample, but now in between two Mn-dots (p-Ge:REF in figure $1(\mathrm{~b})$ ). It can be seen that the $\mathrm{Cu}$ and $\mathrm{Ni}$ contamination is present in both measurements, while two peaks (Mn-H1 and Mn-H2) are only present under the Mn dot. It is remarkable that no strong contamination was observed in n-type underneath the Mn dot, while measurements in the unshielded region shows $\mathrm{Cu}$ and $\mathrm{Ni}$. These observations were confirmed by minority carrier injection; in the lower half of the band gap the levels introduced by the Mn-impurities are clearly observed while no levels assigned to $\mathrm{Cu}$ and $\mathrm{Ni}$ were present.

The thermal emission rate from the electron trap Mn-E1 as a function of temperature may be written as:

$$
e_{n}(T)=\sigma_{n}(T) v_{n}(T) N_{c}(T) \exp \left(-\frac{\Delta G(T)}{k_{B} T}\right)
$$

where $\sigma_{n}(T)$ is the electron capture cross section, $v_{n}(T)$ the thermal velocity of electrons, $N_{c}(T)$ the effective density of states in the conduction band, and $\Delta G(T)$ the Gibbs free 


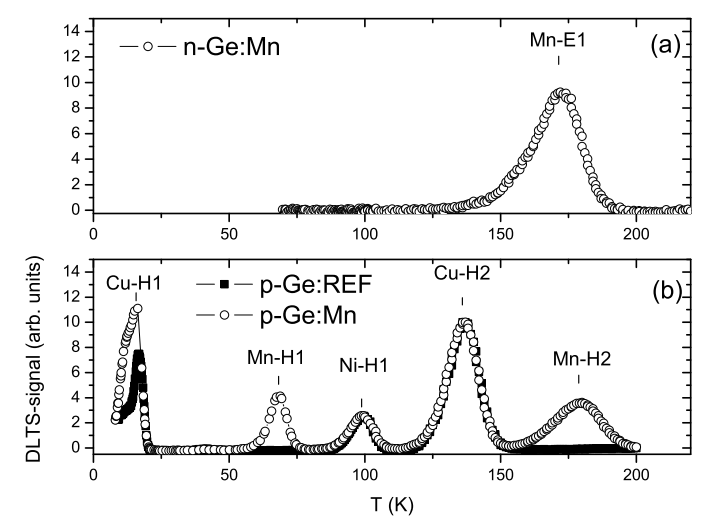

FIG. 1: Conventional temperature DLTS-scan of Ge doped with Mn by indiffusion $t_{\mathrm{w}}=51.2 \mathrm{~ms}$ (a) n-type Ge $\left(\mathrm{V}_{R}=-1 \mathrm{~V}, \mathrm{~V}_{P}=0 \mathrm{~V}\right)$ (b) p-type Ge on the $\mathrm{Mn} \operatorname{dot}\left(\mathrm{p}-\mathrm{Ge}: \mathrm{Mn}, \mathrm{V}_{R}=-3 \mathrm{~V}, \mathrm{~V}_{P}=-2 \mathrm{~V}\right)$ and in between $\mathrm{Mn}$ dots (p-Ge: REF, $\mathrm{V}_{R}=-3 \mathrm{~V}, \mathrm{~V}_{P}=-0.5 \mathrm{~V}$, rescaled).

energy of ionization.[22] As will be shown below, the electron capture cross section of MnE1 exhibits thermal activation in agreeement with multiphonon-assisted capture [23, 24], which may be written as

$$
\sigma_{n}(T)=\sigma_{\infty} \exp \left(-\frac{\Delta E_{\sigma}}{k_{B} T}\right)
$$

Since the Gibbs free energy of ionization corresponding with the distance of the energy level to the conduction band can be expressed in terms of and an enthalpy change and an entropy change $\Delta G=\Delta H-T \Delta S$, the emission rate can also be written as:

$$
e_{n}(T)=\sigma_{\infty} v_{n}(T) N_{c}(T) \exp \left(\frac{\Delta S}{k_{B}}\right) \exp \left(-\frac{\Delta H+\Delta E_{\sigma}}{k_{B} T}\right)
$$

Including the specific temperature dependence of the thermal velocity $v_{n} \propto \sqrt{T}$ and the density of states of the band $N_{c} \propto T^{3 / 2}$, the emission rate can be expressed in a more convenient form for DLTS analysis:

$$
e_{n}(T)=K_{T} T^{2} \exp \left(-\frac{\Delta E_{n a}}{k_{B} T}\right)
$$

The pre-exponential factor $K_{T}$ and the apparant activation energy $\Delta E_{n a}$ form the signature of the deep level. This signature may simply be extracted from an Arrhenius diagram of the experimental emission rates.

The Arrhenius diagram of the levels Mn-E1, Mn-H1 and Mn-H2 is shown in figure 2. 


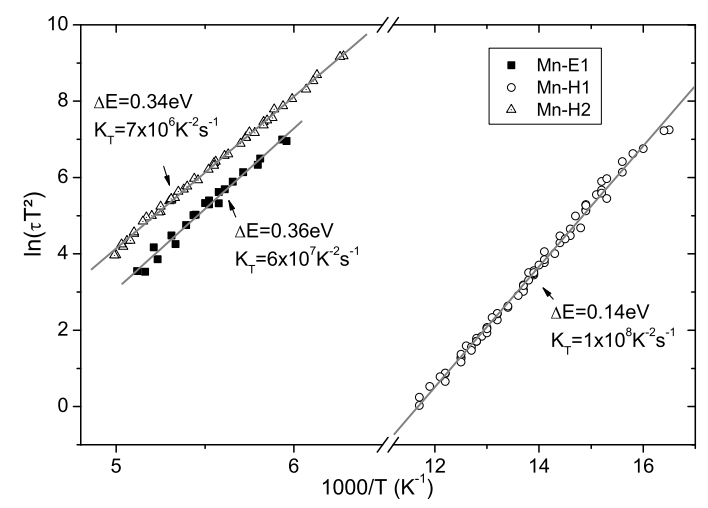

FIG. 2: Arrhenius diagram for the electronic levels assigned to Mn impurities.

The corresponding pre-exponential factor $K_{T}$ and the apparent activation energy $\Delta E_{n a / p a}$ are listed in table I.

The apparent capture cross section is related to the true cross section by

$$
\sigma_{n a / p a}=\sigma_{\infty} \exp \left(\frac{\Delta S}{k_{B}}\right)
$$

where the apparent capture cross section $\sigma_{n a}$ is proportional to $K_{T}$.

The true capture cross section has been measured from the pulse duration dependence of the capacitance signal, as illustrated in figure 3(a) for the Mn-E1 level. The fit to the experimental capacitance transient amplitude as a function of pulse duration corresponds with the procedure described by Lauwaert et al. [25] This procedure based on an analytic approximation taking into account the effect of slow capture at the free carrier tail at the edge of the depletion layer and allowing for a defect concentration profile that was also succesfully used for other transition metal impurities in germanium [26]. From this simulation, a capture cross section of $7.7 \times 10^{-19} \mathrm{~cm}^{2}$ could be extracted. Repeating this measurement for different temperatures showed an exponential dependence of the capture cross section on the temperature. This is illustrated in figure 3(b). From this Arrhenius plot the capture barrier $\Delta E_{\sigma}$ can be determined, being $0.068 \mathrm{eV}$, yielding an activation enthalpy of the level: $\Delta H=\Delta E_{n a}-\Delta E_{\sigma}=295 \mathrm{meV}$. The entropy of this level can be calculated from the true and apparent capture cross section using Eq. (5). The deep level parameters for the different defect levels can be found in table I. The activated capture behavior and the low value of the electron capture cross section are in very good agreement with capture into a negative 

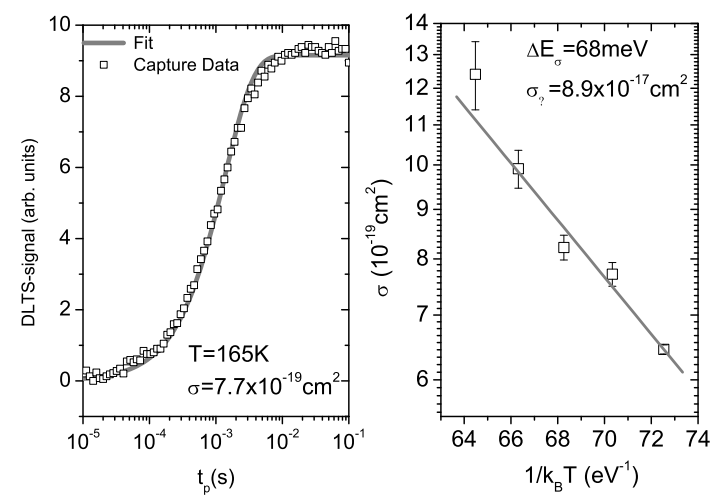

FIG. 3: (a) Direct capture measurement at $165 \mathrm{~K}$ and (b) Arrhenius diagram for the capture cross-section of the $\mathrm{Mn}-\mathrm{E} 1$ level.

charge state, which is the case for multiple acceptors.

To study the charge state of the Mn-ion, EPR measurements were performed at n-Ge:Mn under the following conditions: $\mathrm{T}=4.75 \pm 0.25 \mathrm{~K}, 0.5 \mathrm{~mW}$ microwave power, $100 \mathrm{kHz}$ modulation frequency and 0.4mT modulation-amplitude.[15] The observed spectrum is compatible with the spin Hamiltonian parameters for $\mathrm{Mn}_{\mathrm{s}}^{2-}$ in Ge, reported by Watkins.[15]

\section{DISCUSSION}

Early resistivity measurements performed by Woodbury and Tyler[14], suggest that Mn induces two defect levels in Ge: one in the lower half of the band gap and a second, much deeper, level in the upper half. In DLTS measurements however, three Mn-related defects were observed: two in the lower half of the band gap and a single deep level in the upper half. The activation enthalpy for the Mn-E1 level $(\Delta \mathrm{H}=295 \mathrm{meV})$ corresponds reasonably with the level measured in resistivity measurements on $\mathrm{n}-\mathrm{Ge}: \mathrm{Mn}\left(\mathrm{E}_{\mathrm{C}}-0.37 \mathrm{eV}\right)$, while the Mn-H1 $\left(\mathrm{E}_{\mathrm{V}}+136 \mathrm{meV}\right)$ level corresponds with the level measured in p-Ge:Mn by Hall and resistivity measurements $\left(\mathrm{E}_{\mathrm{V}}+0.16 \mathrm{meV}\right)$. The similar amplitude and depth profile of the Mn-H1 and Mn-H2 peaks suggest that both these levels arise from the same defect, most likely the defect observed in melt-doped Ge.

Because the concentration of other impurities is low, the probability that the Mn-related defect consists of clusters of multiple impurities (e.g. $\mathrm{Mn}-\mathrm{Cu}, \mathrm{Mn}-\mathrm{Ni}$, etc.) is very low. 
Therefore, the two most suitable candidates for the Mn-related defect are interstitial and substitutional Mn. Interstitial TMs are expected to yield donor levels[21], while in substitutional positions TMs give rise to acceptor levels. For the Mn-E1-level, thermally activated capture was observed, this level is repulsive for electrons, thus negatively charged before electron capture and by consequence at least a double acceptor level. The EPR results strongly indicate that the Mn-E1 level corresponds to a $-/ 2$ - charge state transition. Indeed, the EPR spectrum is only observed in n-type Ge:Mn at low temperature, where the Fermi level lies above the $\mathrm{Mn}-\mathrm{E} 1$ level. The assignment of the EPR spectrum to the $\mathrm{Mn}^{2-}$ defect charge state then fixes the lowest valence state of Mn in the Ge band gap. The Mn-H2 and Mn-H1 levels should then be assigned to the $0 /-$ and $+/ 0$ transition respectively. ThisThe assignment is supported by the apparent absence of a Poole-Frenkel shift for all three peaks. Indeed, if both Mn-H1 and Mn-H2 were acceptors, the Mn - H2 level would correspond with the $-/ 2-$ Mn substitutional level. For this level, one expects to see a clear electric field dependence, which is definitely not the case.

Finally, the resemblance between Ge:Co[9] and Ge:Mn should be remarked. Co and Mn both have an odd number of electrons in their partially filled $3 \mathrm{~d}$ shell, and 2 valence selectrons. (Mn: $[\mathrm{Ar}] 3 \mathrm{~d}^{5} 4 \mathrm{~s}^{2}$ and $\mathrm{Co}:[\mathrm{Ar}] 3 \mathrm{~d}^{7} 4 \mathrm{~s}^{2}$ ), they both introduce one donor level and two acceptor levels in the band gap of Ge. It is remarkable that not only $\mathrm{Ge}_{1-\mathrm{x}} \mathrm{Mn}_{\mathrm{x}}$ alloys exhibit ferromagnetic behavior, but also weak ferromagnetic properties were observed for cobalt-germanium compounds[27]. Because also Sc and V have similar electron configurations (Sc: $[\mathrm{Ar}] 3 \mathrm{~d}^{1} 4 \mathrm{~s}^{2}$ and $\mathrm{V}:[\mathrm{Ar}] 3 \mathrm{~d}^{3} 4 \mathrm{~s}^{2}$ ), it seems interesting to check not only whether these impurities also form a donor-level and two acceptor levels in the band gap but also if Sc-Ge and V-Ge can form compounds that exhibit ferromagnetic behavior with potential interest for spintronic devices.

\section{CONCLUSIONS}

DLTS experiments revealed three Mn-related defect levels in Ge: Mn-E1 $\left(\mathrm{E}_{\mathrm{V}}+136 \mathrm{meV}\right)$, $\mathrm{Mn}-\mathrm{H} 2\left(\mathrm{E}_{\mathrm{V}}+342 \mathrm{meV}\right)$ and $\mathrm{Mn}-\mathrm{E} 1\left(\mathrm{E}_{\mathrm{C}}-295 \mathrm{meV}\right)$. These we assign to the $+/ 0,0 /-$ and -/2- levels of substitutional Mn respectively. Woodbury et al. [14] reported two acceptor levels in $\mathrm{Ge}: \mathrm{Mn}$, at positions $\mathrm{E}_{\mathrm{V}}+0.16 \mathrm{eV}$ and $\mathrm{E}_{\mathrm{C}}-0.37 \mathrm{eV}$. These levels correspond reasonably with the $\mathrm{Mn}-\mathrm{H} 1$ and $\mathrm{Mn}-\mathrm{E} 1$ level presented here. However, our experiments 
show that the Mn - H1 level is in fact a donor level and that an additional acceptor level is present: $\mathrm{Mn}-\mathrm{H} 2$. While metal contamination (especially $\mathrm{Cu}$ ) is present in thermally treated samples, it appears that their presence is reduced in $\mathrm{n}-\mathrm{Ge}: \mathrm{Mn}$, indicating that $\mathrm{Mn}$ can shield (or can getter) the contamination.

\section{Acknowledgments}

The authors would like to thank the FWO for financial support through grant no G.0207.10N.

[1] G. Impellizzeri, S. Boninelli, F. Priolo, E. Napolitani, C. Spinella, A. Chroneos and H. Bracht. J. Appl. Phys. 109, 113527 (2011)

[2] R. Kube, H. Bracht, A. Chroneos, M. Posselt and B. Schmidt. J. Appl. Phys. 106, 063534 (2009)

[3] A. Chroneos and H. Bracht, Appl. Phys. Rev. 1, 011301 (2014)

[4] Y.D. Park, A.T. Hanbiki, S.C. Erwin, C.S. Hellberg, J.M. Sullivan, J.E. Mattson, T.F. Ambrose, A. Wilson, G. Spanos, and B.T. Jonker, Science 295, 651 (2002)

[5] Y.F. Tian, J.X. Deng, S.S. Yan, Y.Y. Dai, M.W. Zhao, Y.X. Chen, G.L. Liu, L.M. Mei, Z. Y. Liu, and J.R. Sun, J. Appl. Phys. 107, 024514 (2010)

[6] O. Abbes, A. Portavoce, V. Le Thanh, C. Girardeaux, and L. Michez, Appl. Phys. Lett. 103, $172405(2013)$

[7] N. Sircar, S. Ahlres,C. Majer, G. Abstreiter, and D. Bougeard, Phys. Rev. B. 83, 125306 (2011)

[8] D.V. Lang, J. Appl. Phys. 45, 3023 (1974).

[9] J. Lauwaert, J. Vanhellemont, E. Simoen, H. Vrielinck and P. Clauws, J. Appl. Phys. 111, $113713(2012)$

[10] J. Lauwaert, J. Van Gheluwe, J. Vanhellemont, E. Simoen and P. Clauws, J. Appl. Phys. 105, $073707(2009)$

[11] G. Huylebroeck and P. Clauws and E. Simoen and J. Vennik, Solid State Commun. 82, 367 (1992) 
[12] E. Simoen, P. Clauws, M. Lamon, and J. Vennik, Semicond. Sci. Technol. 1, 53 (1986)

[13] P. Clauws, G. Huylebroeck, E. Simoen, P. Vermaercke, and F. De Smet and J. Vennik, Semicond. Sci. Technol. 4, 910 (1989)

[14] H. H. Woodbury and W. W. Tyler, Phys. Rev. 100, 659 (1955)

[15] G.D. Watkins, Bull. Am. Phys. Soc 2, 235 (1957)

[16] S. Decoster,S. Cottenier, U. Wahl, J.G. Correia, L.M.C. Pereira, C. Lacasta, M.R. Da Silva and A.Vantomme, Appl. Phys. Lett. 97, 151914 (2010)

[17] L. Ottavio, A. Verna, V. Grossi, P. Parisse, S. Piperno, M. Passacantando, G. Impellizeri and F. Priolo, Surface Science 601, 2623-2627 (2007)

[18] S. Zhou and H. Schmidt, Materials 3, 5054 (2010)

[19] E. Simoen, P. Clauws, M. Lamon and J. Vennik, Semicond. Sci. Technol. 1, 53 (1986)

[20] P. Clauws, G. Huylebroeck, E. Simoen, P. Vermaercke, F. De Smet and J. Vennik, Semicond. Sci. Technol. 4, 910 (1989)

[21] C. Claeys and E. Simoen, Germanium-based technologies, Elsevier (2007)

[22] P. Blood and J.W. Orton, 'The electrical characterization of semiconductors: majority carriers and electron states', Academic Press (1992).

[23] D.V. Lang and L.C. Kimerling, Phys. Rev. Lett. 33, 489 (1974)

[24] L.C. Kimerling, Solid-State Electron. 21, 1391 (1978)

[25] J. Lauwaert, J. Van Gheluwe and P. Clauws, Rev. Sci. Instrum. 79, 093902 (2008)

[26] J. Lauwaert and P. Clauws Thin solid films. 518(9), 2330-2333 (2010)

[27] G. S. Patrina, V. K. Maltseva, I. N. Krayukhina, and I. A. Turpanova, Journal of Experimental and Theoretical Physics 117(6), 10971100 (2013) 


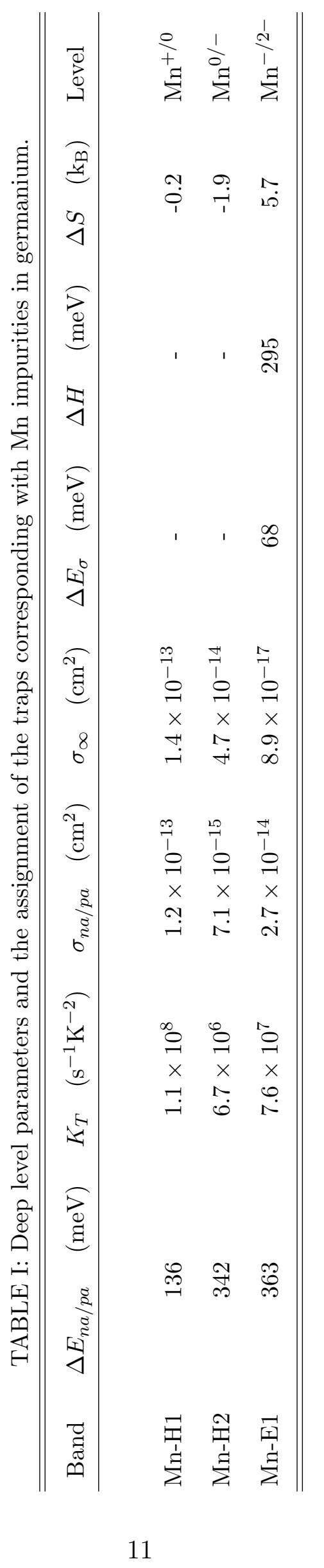

